\title{
ON A CONJECTURE OF CHABAUTY
}

\author{
S. P. WANG
}

Introduction. Let $G$ be a connected Lie group. By a lattice of $G$, we mean a discrete subgroup $\Gamma$ of $G$ such that $G / \Gamma$ has a finite invariant measure. The set of all lattices of $G$ is denoted by $\delta(G)$. In [1], Chabauty introduced the notion of limit of subgroups of $G$. A sequence $\left\{H_{n}\right\}$ of subgroups of $G$ converges to a subgroup $H$ if for any given compact subset $K$ of $G$ and neighborhood $V$ of the identity $e$ in $G, H \cap K \subset V H_{n}$ and $H_{n} \cap K \subset V H$ hold for sufficiently large $n$. Thus $\delta(G)$ becomes a topological space with the Chabauty topology defined by limit of lattices. In [5], some topological properties of $\S(G)$ have been studied. $\delta(G)$ is separable metric. However in general we do not know whether $\delta(G)$ is locally compact or not. Let $A(G)$ be the group of all continuous automorphisms of $G$. Equipped with the compact-open topology, $A(G)$ is a Lie group. $A(G)$ operates continuously on $\delta(G)$ with operation defined by $(\alpha, \Gamma) \rightarrow \alpha(\Gamma)$, for $\alpha \in A(G)$ and $\Gamma \in S(G)$. In [1], Chabauty conjectured that for any lattice $\Gamma$ of $G, A(G) \Gamma$ with induced topology from $\delta(G)$ is homeomorphic to $A(G) / N(\Gamma)$, where $N(\Gamma)$ is the isotropy subgroup at $\Gamma$, or equivalently $A(G) \Gamma$ is locally compact. Followed by a theorem of Malcev [3], the conjecture is true for nilpotent Lie groups. For semisimple Lie groups, the author obtained some partial results in [5]. The purpose of this paper is to construct a counterexample in the case of solvable Lie groups.

1. Semidirect product of a compact group and a vector group. Let $V=R^{n}$ and $K$ a compact subgroup of $\mathrm{GL}(n, R)$. In $G=K \times V$ (in the sense of set only), we define a group structure by

$$
(k, v)\left(k_{1}, v_{1}\right)=\left(k k_{1}, k_{1}^{-1} v+v_{1}\right)
$$

for $k, k_{1} \in K$ and $v, v_{1} \in V$.

Leмма 2. $V$ is the nilpotent radical of $G$, i.e., the maximal connected normal nilpotent subgroup of $G$.

Proof. Let $n(G)$ be the nilpotent radical of $G$. Clearly $n(G) \supset V$. Hence $n(G)=(n(G) \cap K) \cdot V$. Since $n(G) \cap K$ is compact, it is central in $n(G)$. However the action of $K$ on $V$ is faithful. It implies that $n(G) \cap K=\{e\}$. Thus $V=n(G)$.

Received by the editors February 20, 1969. 
As $V$ is a characteristic subgroup of $G$, we have then the restriction map res: $A(G) \rightarrow \mathrm{GL}(V)$ and the induced map ind: $A(G) \rightarrow A(K)$. Let $C(K)$ be the centralizer of $K$ in $\mathrm{GL}(n, R)$ and $A(G)^{0}$ the identity component of $A(G)$.

Lemma 3. If $K$ is commutative, then $\operatorname{res}\left(A(G)^{0}\right)=C(K)^{0}$.

Proof. Since $K$ is compact and abelian, it is well known $A(K)^{0}$ $=\{e\}$. Hence ind $\left(A(G)^{0}\right)=\{e\}$. Clearly

$$
\begin{aligned}
(e,(\alpha k) x) & =\alpha((e, k x))=\alpha\left((k, e)(e, x)(k, e)^{-1}\right) \\
& =(k, e) \alpha((e, x))(k, e)^{-1}=(e,(k \alpha) x)
\end{aligned}
$$

for all $\alpha \in A(G)^{0}, k \in K, x \in V$. Therefore $\operatorname{res}\left(A(G)^{0}\right) \subset C(K)^{0}$. Conversely given any $\beta \in C(K)$, we define $\tilde{\beta}((k, x))=(k, \beta x),(k, x) \in G$. It is obvious that $\tilde{\beta} \in A(G)$ and $\operatorname{res}(\widetilde{\beta})=\beta$. Thus $\operatorname{res}\left(A(G)^{0}\right)=C(K)^{0}$.

4. Diophantine approximation. Let $\gamma$ be an irrational number. As an immediate consequence of diophantine approximation, there exists an increasing sequence $\left(a_{n}\right)$ of positive integers such that $\left(a_{n} \gamma-\left[a_{n} \gamma\right]\right)$ converges to zero, where $[x]$ is the function of the greatest integer $\leqq x, x \in R$. Denote $\left[a_{n} \gamma\right]$ and $a_{n} \gamma-\left[a_{n} \gamma\right]$ by $b_{n}$ and $c_{n}$ respectively. By an easy computation, we have

$$
\begin{aligned}
*\left(\begin{array}{cccc}
1 & 0 & 0 & 0 \\
0 & 1 & 0 & 0 \\
0 & 0 & 1+a_{n}-a_{n} \\
0 & 0 & -1 & 1
\end{array}\right)\left(\begin{array}{cccc}
1 & 0 & \gamma & 0 \\
0 & 1 & 0 & 0 \\
0 & 0 & 1 & 0 \\
0 & 0 & 0 & 1
\end{array}\right)\left(\begin{array}{cccc}
1 & 0 & 0 & -b_{n} \\
0 & 1 & 0 & 0 \\
0 & 0 & 1 & a_{n} \\
0 & 0 & 1 & 1+a_{n}
\end{array}\right) \\
=\left(\begin{array}{cccc}
1 & 0 & \gamma & c_{n} \\
0 & 1 & 0 & 0 \\
0 & 0 & 1 & 0 \\
0 & 0 & 0 & 1
\end{array}\right) .
\end{aligned}
$$

Let $Q$ be the subgroup of $\mathrm{GL}(4, R)$ containing all matrices of the form

$$
\left(\begin{array}{cccc}
\cos \theta & \sin \theta & 0 & 0 \\
-\sin \theta & \cos \theta & 0 & 0 \\
0 & 0 & 1 & 0 \\
0 & 0 & 0 & 1
\end{array}\right) \quad \theta \in R .
$$

Then the centralizer $C(Q)$ of $Q$ in $\operatorname{GL}(4, R)$ is the subgroup of $\mathrm{GL}(4, R)$ consisting of all matrices of the form 


$$
\left(\begin{array}{llll}
\lambda & 0 & 0 & 0 \\
0 & \lambda & 0 & 0 \\
0 & 0 & & \\
0 & 0 & X &
\end{array}\right) \quad \lambda \in R-\{0\} \text { and } X \in \mathrm{GL}(2, R)
$$

Let

$$
\bar{\gamma}=\left(\begin{array}{llll}
1 & 0 & \gamma & 0 \\
0 & 1 & 0 & 0 \\
0 & 0 & 1 & 0 \\
0 & 0 & 0 & 1
\end{array}\right) \text { and } \bar{\gamma}_{n}=\left(\begin{array}{llll}
1 & 0 & \gamma & c_{n} \\
0 & 1 & 0 & 0 \\
0 & 0 & 1 & 0 \\
0 & 0 & 0 & 1
\end{array}\right) .
$$

Due to our construction, $\bar{\gamma}_{n} \rightarrow \bar{\gamma}$ and $\bar{\gamma}_{n} \neq \bar{\gamma}_{m}$ for $n \neq m$. It is very easy to see that

$$
C(Q) \gamma_{n} \neq C(Q) \gamma_{m} \quad \text { for } n \neq m .
$$

Proposition 5. $C(Q)^{0} \bar{\gamma} \operatorname{SL}(4, Z)=\left\{x \bar{\gamma} y \mid x \in C(Q)^{0}\right.$ and $\left.y \in \operatorname{SL}(4, R)\right\}$ is not locally closed.

Proof. Suppose false. Then $C(Q)^{0} \bar{\gamma}$ is open in $C(Q)^{0} \bar{\gamma} \operatorname{SL}(4, Z)$ and $\bar{\gamma}_{n} \rightarrow \bar{\gamma}$. This implies $C(Q)^{0} \bar{\gamma}_{n}=C(Q)^{0} \bar{\gamma}$ for sufficiently large $n$. However this contradicts the fact that $C(Q) \bar{\gamma}_{n} \neq C(Q) \bar{\gamma}_{m}$ for $n \neq m$.

6. A counterexample. Let $V=R^{4}, K=\bar{\gamma}^{-1} Q \bar{\gamma}, G=K \cdot V$ as constructed in $\S 1$, and $\Gamma=Z^{4} \subset R^{4}$. Since $G / \Gamma$ is compact, certainly $\Gamma$ is a lattice of $G$.

Main Theorem. $A(G) \Gamma$ is not locally compact with induced topology $\S(G)$.

Proof. Suppose false. Then $A(G)^{\circ} \Gamma$, open in $A(G) \Gamma$ is locally compact. By Lemma 3, $A(G)^{0} \Gamma=\operatorname{res}\left(A(G)^{0}\right) \Gamma=C(K)^{0} \Gamma$. Let $\AA_{v}(G)$ be the set of all lattices of $G$ contained in $V$. It is clear that $\delta_{v}(G)$ is a closed subset of $\delta(G)$ and $\delta_{v}(G)=\delta(V)$. It is a well-known classical result $\delta(V) \approx \mathrm{GL}(V)^{0} / N(\Gamma)$, where $N(\Gamma)=\mathrm{SL}(4, \boldsymbol{Z})$. Therefore it follows that $C(K)^{0} \mathrm{SL}(4, \boldsymbol{Z})$ is locally compact. But $C(K)^{0} \mathrm{SL}(4, \boldsymbol{Z})$ $=\bar{\gamma}^{-1}\left(C(Q)^{0} \bar{\gamma} \mathrm{SL}(4, \boldsymbol{Z})\right)$ is not locally compact by Proposition 5 . Thus we are led to contradiction.

A REMARK. Although Chabauty's conjecture is not true in solvable Lie groups, it is still very likely that the conjecture will be valid in semisimple Lie groups supported by some indications in [5]. If $G$ is semisimple with each factor of $R$-rank $\geq 2, \delta(G)$ is locally compact which is an easy consequence of [7]. 


\section{REFERENCES}

1. C. Chabauty, Limite d'ensembles et gémétrie des nombres, Bull. Soc. Math. France 78 (1950), 143-151.

2. A. M. Macbeath and S. Swiercjkowski, Lattices in a compactly generated group, Canad. J. Math. 7 (1960), 427-438.

3. A. I. Malcev, On a class of homogeneous spaces, Amer. Math. Soc. Transl. (1) 9 (1962), 276-307.

4. H. C. Wang, On the deformation of lattices in a Lie group, Amer. J. Math. 85 (1963), 180-212.

5. S. P. Wang, Limit of lattices in a Lie group, Trans. Amer. Math. Soc. 133 (1968), 519-526.

6. - The dual space of semisimple Lie groups, Amer. J. Math. (to appear).

7. - On a theorem of representation of lattices, Proc. Amer. Math. Soc. (to appear).

Institute for Advanced Study 